\title{
Improving inhaler use in COPD and the role of patient preference
}

\author{
E.D. Bateman
}

ABSTRACT: Chronic obstructive pulmonary disease (COPD) is a difficult disease to manage, but recent research focusing on its pathophysiology has provided direction for the development of new treatments and improved management strategies.

COPD differs substantially from asthma, both in its pathophysiology and its treatment. Unlike asthma, COPD cannot be fully controlled or "reversed"; it is progressive and responses to antiinflammatory drugs, including corticosteroids, have been disappointing, suggesting the presence of a unique, persistent form of inflammation. The current main emphasis in the treatment of COPD is therefore to minimise airflow obstruction using regular bronchodilator therapy. Apparently small improvements in traditional measures of airflow obstruction, such as functional residual capacity, may produce significant improvements in other measures, resulting in clinical benefit.

Ensuring efficient delivery of bronchodilators is vital to treatment success but has received little attention in guidelines to date. Inhaler technique, adherence rates and levels of satisfaction with therapy are all far from ideal. Improvements in these areas require more detailed consideration of the interactions between the patient, the healthcare provider and the inhaler device, and an examination of how inhaler choices are currently made by both healthcare provider and patient.

KEYWORDS: Chronic obstructive pulmonary disease, inhalation devices, patient preference

I $\mathrm{n}$ the spectrum of chronic diseases, chronic obstructive pulmonary disease (COPD) presents a number of challenges that make it one of the most difficult to manage. Its insidious and generally asymptomatic onset militates against early detection and secondary prevention. It also occurs in the elderly at a time when they are resigned to a life of restricted activity. This may reduce their demand for effective therapies, lessen their adherence to therapy, and lead to cost compromises at the expense of health. Many elderly patients also find it difficult to use pressurised metered-dose inhalers and other forms of inhalation therapy [1-3]. Additional factors in COPD, which do not appear to influence the care of patients with coronary artery disease and other forms of smokingrelated illnesses, are the emotional and even subconscious influences on the patient and carer of managing what is considered to be a selfinflicted disease.

These considerations, together with a lack of effective therapies, account for the "Cinderella" status ascribed to COPD until relatively recently [4]. However, the realisation that mortality from COPD is rising at a time when deaths from other chronic diseases (including coronary artery and cerebrovascular disease) are on the decline, and the widely publicised prediction that COPD is set to move from sixth to third place amongst the most common causes of death globally by the year 2020 [5], have led to renewed interest in this disease.

\section{THE DIFFERING PATHOLOGY OF ASTHMA AND CHRONIC OBSTRUCTIVE PULMONARY DISEASE}

Results of research into the pathophysiology of this disease and the mechanisms responsible for breathlessness in patients with COPD provide the basis for treatment and have identified potential targets for therapy [6-10]. Such work COPD and asthma, and the importance of distinguishing between the two, not only because the pathophysiology underlying these conditions differs, but also because the treatment approach taken to the two diseases is strikingly different [6-8, 10-13].

Asthma cannot be prevented but it can be fully controlled with current treatments, permitting those affected to live normal symptom-free lives for significant periods of time [12]. In contrast, COPD is fully preventable but cannot be fully reversed, and may progress (causing deterioration) over time [13]. The inflammatory process in has also highlighted the differences between
CORRESPONDENCE

E.D. Bateman

UCT Lung Institute

P0 Box 34560

7937 Groote Schuur

Cape Town

South Africa

Fax: 27214066902

E-mail:

ebateman@uctgsh1.uct.ac.za 
airways in asthma is dominated by eosinophils, mast cells and CD4+ lymphocytes, with the major mediators of tissue damage being leukotriene (LT)D4, histamine, interleukin (IL)-4 and IL5 [12]. In COPD, the number of neutrophils and CD8+ cells is increased and LTB4, histamine, IL-8 and tumour necrosis factor (TNF)- $\alpha$ play a leading role in the disease $[8,13]$. In terms of pathology, chronic changes in asthmatic airways include shedding of epithelium and thickened subepithelial tissues, comprising a fibrotic lamina propria, increased vascularity and hypertrophy of smooth muscle. Although inflammation and fibrosis are found in submucosal tissues in COPD, and particularly in small airways, disruption of the architecture of acini results from loss of elastic tissue in alveolar walls and loss of alveolar attachments. In larger airways, mucus hypersecretion and squamous metaplasia are common features [7]. As a result of these differences, the treatment approach to the diseases differs.

\section{TREATMENT APPROACHES}

In asthma, the primary concern is to address the airway inflammation through use of inhaled corticosteroids and other controller medications, thereby reducing airway hyperresponsiveness and achieving control of symptoms. With this approach, the aim is to reduce bronchodilator use (particularly for the treatment of breakthrough symptoms) to a minimum, so that the controlled patient can spend long periods of time without the need for rescue medication [12]. By contrast, in COPD no anti-inflammatory drug has been identified that has an equivalent effect to inhaled corticosteroids in asthma; these agents have relatively little effect in COPD [13, 14]. Instead, the strategy is to address the mechanisms of airflow obstruction and to attempt to improve or optimise function [9, 10, 14, 15].

A variety of mechanisms account for airflow obstruction in COPD. Irreversible obstruction is a result of fibrosis and narrowing of the airways, loss of elastic recoil due to alveolar destruction, and depletion of the alveolar support that keeps small airways open throughout the respiratory cycle, including during forced expiration. The last of these mechanisms causes the dynamic collapse of intrathoracic airways characteristic of emphysematous lung, and is aggravated by rapid breathing occurring during exertion or excitement [9]. Reversible airflow limitation is caused by an element of airway hyperresponsiveness found in the majority of patients with advanced disease, and is often associated with a small but reproducible degree of increased bronchoconstrictor tone (usually mediated by the cholinergic parasympathetic nervous system) [9]. These reversible elements in COPD account for most of the improvement in forced expiratory volume in one second (FEV1) observed in this disease. However, additional benefits are gained from the changes in other volumes of the lung achieved with a bronchodilator. For example, O'DoNNELL et al. [16] demonstrated that the reduction of the functional residual capacity at rest with tiotropium, a long-acting once-daily anticholinergic agent, results in improvement in inspiratory capacity and exercise capacity in patients with severe COPD.

Thus, seemingly small improvements in traditional measures of airflow obstruction may be associated with significant improvements in other parameters, resulting in clinical benefit [14]. This forms the basis of the recommendations on bronchodilator usage given in the guidelines of the Global Initiative for Chronic Obstructive Lung Disease (GOLD), namely that bronchodilators are central to the symptomatic management of COPD and that as symptoms increase, they are best given as a regular dose [13]. Incremental dosing is based on symptoms and may involve the use of higher doses of the bronchodilator, or the addition of a second or third bronchodilator, with options being a long-acting $\beta_{2}$-agonist, an inhaled anticholinergic drug and/or theophylline. This approach improves efficacy while limiting the potential for adverse effects of drugs. Used in this manner, bronchodilators have been shown to reduce symptoms, prevent exacerbations, and improve both health status and exercise capability $[13,15,16]$.

\section{CONSIDERATIONS IN DRUG DELIVERY}

Sustained bronchodilatation provided by the regular use of long-acting bronchodilators is therefore the best treatment option for symptomatic patients with COPD. The next challenge is to ensure that drug delivery to the lung is efficient. For a variety of reasons, this is more difficult than it sounds, particularly in patients with COPD. The three

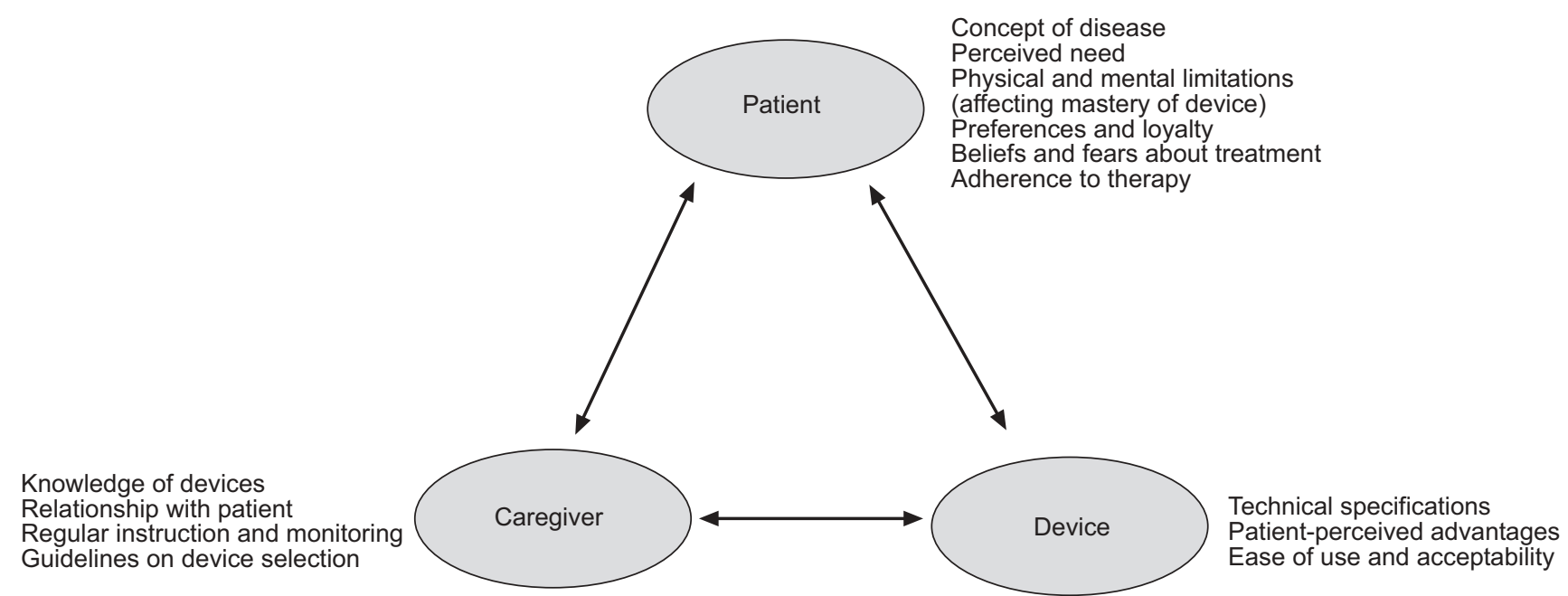

FIGURE 1. The triangle of influences on inhaler-device usage by patients with chronic obstructive pulmonary disease. 
important factors in drug delivery (fig. 1) are the patient, the inhalation device and the provider; together, these influence whether optimal delivery of the bronchodilator will be achieved. These factors and the interactions between them are considered in more detail in the papers that follow.

Considering the obvious importance of drug delivery to the success of treatment, it is perhaps surprising that the issues surrounding it have attracted so little attention in treatment guidelines. The GOLD guidelines contain little advice on delivery devices and their performance, inhaler selection, methods for improving compliance and the impact of patient preference on drug delivery and treatment outcomes [13]. Yet even superficial scrutiny of published literature reveals a number of concerning issues: low levels of adherence to regular use of bronchodilators, deliberate patient falsification of information on inhaler use (suggesting complicated and unsatisfactory relationships between patients and carers) [17], poor technique in the use of pressurised metered-dose inhalers (the most widely used device in patients with COPD) [2], and low levels of satisfaction with the effects of treatment [18], to name but a few. It is therefore appropriate to consider the topic in some detail and to define the questions that need to be addressed. With the COPD patient in mind, the following need to be considered:

- Influence of patient preference and choice. What influence does patient choice of the inhaler device have upon treatment adherence and clinical outcomes? Is it important to provide choices and respond to the patient's preference and wishes, or should other factors take precedence in the selection of a device? How does the patient's personality phenotype influence adherence and how should this affect the plan of treatment? Does one approach suit all patients?

- Influence of performance characteristics of inhalation devices. What do we know about the performance of different inhalation devices, and how might these characteristics influence treatment choice and adherence?

- Role of the caregiver. What should the role of the caregiver be in enhancing drug delivery? What should their role be in providing a selection of suitable devices to enable each patient to exercise choice? Do doctors currently take preferences expressed by patients into account?

The study of patients and their interactions with carers and treatment devices in chronic obstructive pulmonary disease has lagged behind that of the more technical aspects of inhaler design. While new designs, like some of the multi-dose dry powder inhalers and the Respimat ${ }^{\circledR}$ Soft Mist ${ }^{\mathrm{TM}}$ Inhaler (Boehringer Ingelheim $\mathrm{GmbH} \& \mathrm{Co}$. KG, Ingelheim, Germany), offer reliable and superior dosing [19, 20], less is known about the advantages of these developments in "real

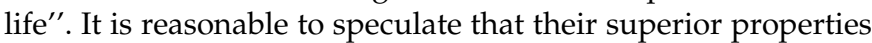
might be of particular value in subjects who fail to be included in clinical trials (by virtue of their less-than-optimal handling of devices), but more research of this nature is required. Until the results of such trials are available, the advice for clinicians is to: achieve and maintain open communication about inhalational treatment with patients; be aware of differences in devices that might influence both drug delivery and adherence; individualise inhaler choice to accommodate needs, fears and preferences of patients; and monitor both the clinical outcomes and the concordance of patients with intended treatment.

\section{SUMMARY}

- The pathophysiology of chronic obstructive pulmonary disease and asthma differ, and require different treatment approaches.

- Maintenance of sustained bronchodilation has been shown to improve several key clinical and physiological features of disease.

- The management of chronic obstructive pulmonary disease presents challenges relating to the age and expectations of patients, and their readiness to use and ability to handle inhaled drugs.

- Ensuring efficient delivery of drugs in chronic obstructive pulmonary disease patients is vital to treatment success but has received little attention.

- The three key factors in ensuring effective inhalation therapy are: the characteristics of the inhalation device; the patient's knowledge, attitudes and preference; and the physician's familiarity with inhalers and their skill in understanding the patient's needs and preferences. Together, these factors influence satisfaction with therapy, which may affect longterm adherence and clinical outcomes.

\section{REFERENCES}

1 Molimard M, Raherison C, Lignot S, Depont F, Abouelfath A, Moore N. Assessment of handling of inhaler devices in real life: an observational study in 3811 patients in primary care. J Aerosol Med 2003; 16: 249-254.

2 Armitage JM, Williams SJ. Inhaler technique in the elderly. Age Ageing 1988; 17: 275-278.

3 Jones V, Fernandez C, Diggory P. A comparison of large volume spacer, breath-activated and dry powder inhalers in older people. Age Ageing 1999; 28: 481-484.

4 Fishman AP. One hundred years of chronic obstructive pulmonary disease. Am J Respir Crit Care Med 2005; 171 941-948.

5 Murray CJ, Lopez AD. Alternative projections of mortality and disability by cause 1990-2020: Global Burden of Disease Study. Lancet 1997; 349: 1498-1504.

6 Saetta M, Di Stefano A, Turato G, et al. CD8+ Tlymphocytes in peripheral airways of smokers with chronic obstructive pulmonary disease. Am J Respir Crit Care Med 1998; 157: 822-826.

7 Baraldo S, Saetta M, Cosio MG. Pathophysiology of the small airways. Semin Respir Crit Care Med 2003; 24: 465-472.

8 Barnes PJ. Mediators of chronic obstructive pulmonary disease. Pharmacol Rev 2004; 56: 515-548.

9 O'Donnell DE, Forkert L, Webb KA. Evaluation of bronchodilator responses in patients with "irreversible" emphysema. Eur Respir J 2001; 18: 914-920.

10 Liesker JJ, Wijkstra PJ, Ten Hacken NH, et al. A systematic review of the effects of bronchodilators on exercise capacity in patients with COPD. Chest 2002; 121: 597-608.

11 Shapiro SD. COPD unwound. N Engl J Med 2005; 352: 2016-2019. 
12 Global Initiative for Asthma. Global strategy for asthma management and prevention. NHLBI/WHO Workshop Report. Publication No.02-3659. Bethesda, National Institutes of Health, National Heart Lung and Blood Institute, 2002.

13 Global Initiative for Chronic Obstructive Lung Disease (GOLD). Global strategy for the diagnosis, management, and prevention of chronic obstructive pulmonary disease. NHLBI/WHO Workshop Report. Bethesda, National Institute of Health, National Heart, Lung, and Blood Institute, publication updated 2003.

14 Calverley PM. The role of corticosteroids in chronic obstructive pulmonary disease. Semin Respir Crit Care Med 2005; 26: 235-245.

15 Sin DD, McAlister FA, Man SF, Anthonisen NR. Contemporary management of chronic obstructive pulmonary disease: scientific review. JAMA 2003; 290: 2301-2312.
16 O'Donnell DE, Fluge T, Gerken F, et al. Effects of tiotropium on lung hyperinflation, dyspnoea and exercise tolerance in COPD. Eur Respir J 2004; 23: 832-840.

17 Rand CS, Nides M, Cowles MK, Wise RA, Connett J. Longterm metered-dose inhaler adherence in a clinical trial. The Lung Health Study Research Group. Am J Respir Crit Care Med 1995; 152: 580-588.

18 Jones RCM, Hyland ME, Hanney K, Erwin J. A qualitative study of compliance with medication and lifestyle modification in chronic obstructive pulmonary disease (COPD). Prim Care Respir J 2004; 13: 149-154.

19 Kilfeather SA, Ponitz HH, Beck E, et al. Improved delivery of ipratropium bromide/fenoterol from Respimat Soft Mist Inhaler in patients with COPD. Respir Med 2004; 98: 387-397.

20 Keller M. Innovations and perspectives of metered dose inhalers in pulmonary drug delivery. Int J Pharm 1999; 186: 81-90. 